\title{
Surface termination and electronic reconstruction in $\mathrm{YBa}_{2} \mathrm{Cu}_{3} \mathrm{O}_{7-\delta}$
}

\author{
Hideaki Iwasawa, ${ }^{1,}{ }^{*}$ Niels B. M. Schröter,${ }^{2, \dagger}$ Takahiko Masui, ${ }^{3}$ Setsuko Tajima, ${ }^{4}$ Timur K. Kim,,${ }^{1}$ and Moritz Hoesch ${ }^{1,5}$ \\ ${ }^{1}$ Diamond Light Source, Harwell Science and Innovation Campus, Didcot OX11 ODE, United Kingdom \\ ${ }^{2}$ Department of Physics, University of Oxford, Oxford OX1 3PU, United Kingdom \\ ${ }^{3}$ Department of Physics, Kindai University, Higashi-Osaka 577-8502, Japan \\ ${ }^{4}$ Department of Physics, Osaka University, Osaka 560-0043, Japan \\ ${ }^{5}$ Deutsches Elektronen-Synchrotron (DESY), Photon Science, Notkestrasse 85, 22607 Hamburg, Germany
}

(Received 12 July 2018; published 21 August 2018)

\begin{abstract}
We present a spatially resolved angle-resolved photoemission spectroscopy (ARPES) study on the Y-based high- $T_{c}$ cuprate superconductor $\mathrm{YBa}_{2} \mathrm{Cu}_{3} \mathrm{O}_{7-\delta}$ to disentangle surface electronic inhomogeneity. Two surface terminations consisting of either a $\mathrm{CuO}$ or $\mathrm{BaO}$ layer are identified through a chemical-states-specified core-level intensity distribution. This enables us to perform termination-selective ARPES measurements that uncover the different charge fillings and electronic configurations depending on the surface termination. By combining the real-space and electronic information, we propose a simple model to explain the termination-dependent surface electronic reconstruction. Our results demonstrate a significant importance of the spatially resolved ARPES in order to obtain intriguing electronic information, particularly from surface-inhomogeneous systems.
\end{abstract}

DOI: 10.1103/PhysRevB.98.081112

Among high- $T_{c}$ superconducting cuprates, yttrium barium copper oxide, $\mathrm{YBa}_{2} \mathrm{Cu}_{3} \mathrm{O}_{7-\delta}$ (YBCO), is known as a peculiar system possessing not only two-dimensional (2D) $\mathrm{CuO}_{2}$ planes but also quasi-one-dimensional (1D) $\mathrm{CuO}$ chains. This unique crystallographic nature of YBCO has made it challenging to study the electronic structure by angle-resolved photoemission spectroscopy (ARPES) because of the intermixing of multiple surface terminations on the cleaved surfaces. As shown in Fig. 1(a), the bilayer $2 \mathrm{D} \mathrm{CuO}_{2}$ planes with an insertion of the $\mathrm{Y}$ atom are a strongly bonded and stable block, which also attracts the $\mathrm{Ba}$ atom. A cleavage plane can thus be naturally expected to be at the bond between $\mathrm{BaO}-\mathrm{CuO}$ or $\mathrm{CuO}-\mathrm{BaO}$ [dashed lines in Fig. 1(b)], resulting in either $\mathrm{CuO}$ - or $\mathrm{BaO}$-terminated surfaces, as confirmed by scanning tunneling microscopy (STM) experiments [1].

Existing ARPES studies on nearly optimally doped YBCO observed two components [2,3], which have been discussed in terms of a surface-bulk dichotomy, namely, the out-of-plane electronic inhomogeneity. One component, often referred to as bulk states, is assumed to be derived from the subsurface layer which may maintain a nominal doping level [2-5]. The other component, often referred to as surface states, is heavily overdoped and localized at the topmost surface [6,7]. However, this interpretation is rather unrealistic because the distance from the $\mathrm{CuO}$ - and $\mathrm{BaO}$-terminated surfaces to the subsurface bilayer is about 12 and $14 \AA[8]$, respectively, both of which are

\footnotetext{
*Author to whom correspondence and requests for materials should be addressed: h-iwasawa@ hiroshima-u.ac.jp; present address: Graduate School of Science, Hiroshima University, 1-3-1 Kagamiyama, Higashi-Hiroshima, Hiroshima 739-8526, Japan.

${ }^{\dagger}$ Present address: Swiss Light Source, Paul Scherrer Institut, CH5232 Villigen PSI, Switzerland.
}

longer than the electron mean free path $(\sim 4-6 \AA)$ for typically used photon energies $(\sim 20-60 \mathrm{eV})$ [9].

On the other hand, the origin of the surface overdoping was discussed in terms of the surface polarity of YBCO [10]. It was also reported that the doping level on the surface layer is successfully controlled from overdoping to underdoping by depositing potassium atoms in situ [11]. However, the mechanism of surface electronic reconstruction in YBCO has not been fully detailed. Moreover, the understanding of in-plane electronic inhomogeneity is also far from sufficient.

In this Rapid Communication, we present a high-resolution ARPES with sub-hundred-micron spatial resolution (microARPES) study on the cleaved surface of optimally doped YBCO. We reveal surface electronic inhomogeneity accompanied by different chemical states, which are fingerprinted by the chemical shift of the Ba $4 d$ core level. These observations allow us to identify the spatial distribution of the surface terminations, and to unveil the termination-dependent charge dynamics and electronic reconstruction in YBCO. We present a simple model to explain the observed termination-dependent surface electronic reconstruction, which cannot be explained by the prototypical polar catastrophe mechanism.

High-quality single crystals of optimally doped YBCO $\left(\delta=0.1, T_{c}=93 \mathrm{~K}\right)$ were grown by the crystal pulling technique and detwinned by annealing under uniaxial pressure [12]. Micro-ARPES experiments were performed at beamline I05 of the Diamond Light Source using a high-resolution hemispherical electron analyzer (R4000, Scienta) [13]. All the measurements were performed after cleaving the samples in situ under ultrahigh-vacuum conditions $\left(2 \times 10^{-10} \mathrm{mbar}\right)$ at temperatures around $8 \mathrm{~K}$. The energy, angular, and lateral resolution were set to be $5-8 \mathrm{meV}, 0.2^{\circ}$, and $\sim 60 \mu \mathrm{m}$, respectively.

The Fermi surface of YBCO [Fig. 1(d)] is composed of a $1 \mathrm{D} \mathrm{CuO}$-chain band $(\mathrm{CH})$ and a $2 \mathrm{D} \mathrm{CuO}_{2}$-plane band that are 


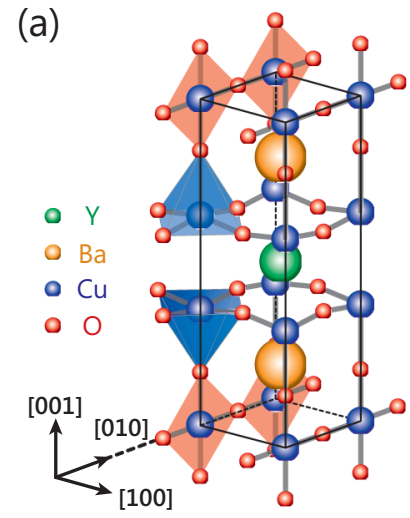

(d)

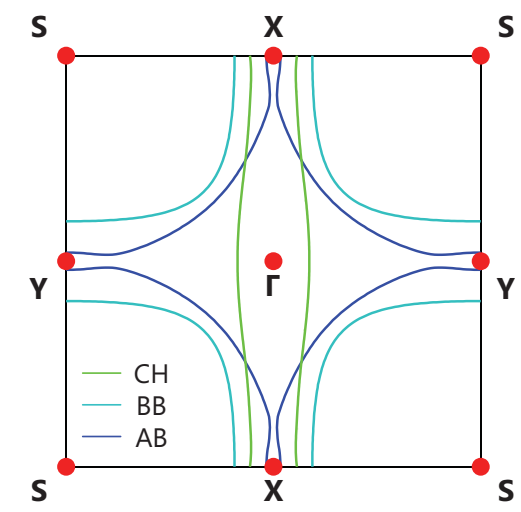

(b)

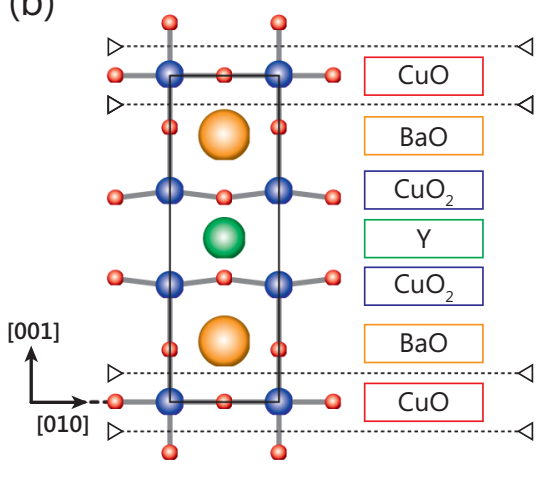

(e)

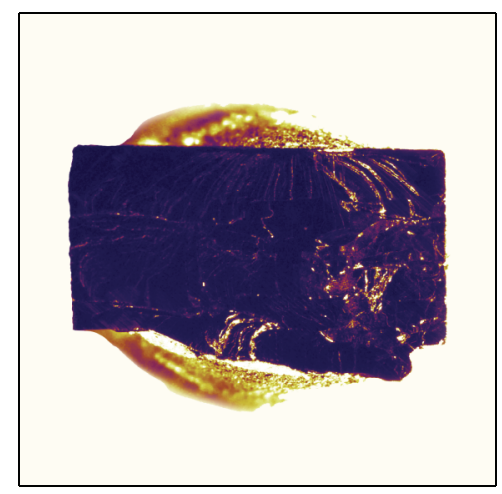

(c)

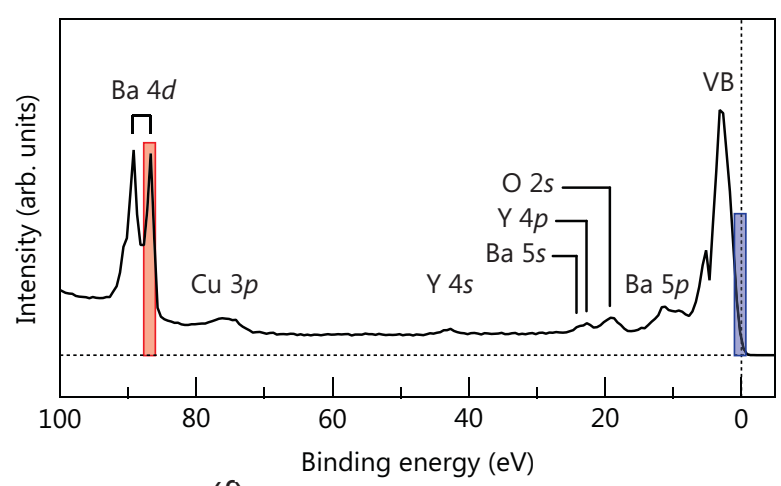

(f)

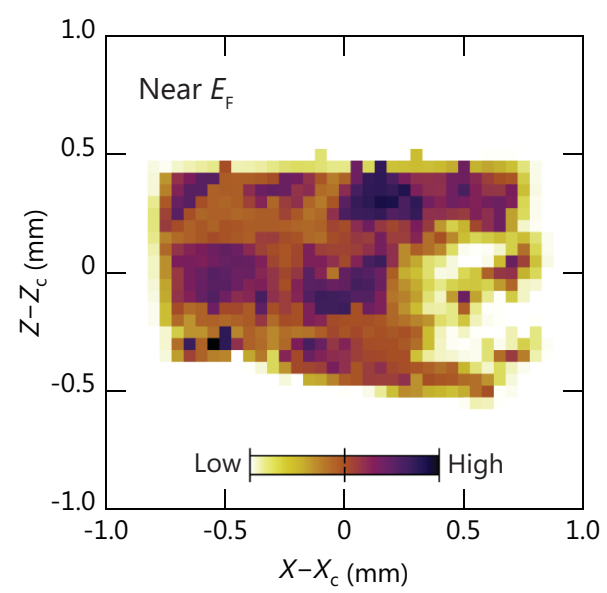

FIG. 1. Overview of ARPES intensity distribution on a cleaved surface of YBCO. The orthorhombic crystal structure of optimally doped YBCO shown in (a) a topdown view and (b) a cross-sectional view, respectively. The crystal is expected to cleave somewhere in between the $\mathrm{CuO}$ and $\mathrm{BaO}$ bonds, as indicated by the horizontal dashed lines in (b). (c) Representative angle-integrated energy distribution curve (EDC). (d) Schematic Fermi surfaces of $\mathrm{YBCO}$ composed of the $\mathrm{CuO}$ chain $(\mathrm{CH})$ and the bonding and antibonding bands of the $\mathrm{CuO}_{2}$ plane $(\mathrm{BB}$ and AB). (e) An optical microscope image taken ex situ after ARPES experiments. (f) ARPES intensity distributions near the Fermi level, whose integration window is indicated by the blue shaded area in (c). Photoemission data are taken at a photon energy of $150 \mathrm{eV}$ below $7 \mathrm{~K}$.

bilayer split into bonding and antibonding bands (BB and $\mathrm{AB}$ ) [10]. Here, we refer to the high-symmetry points as in the 2D Brillouin zone (BZ) and not in the 3D BZ for an orthorhombic crystal structure following the convention for simplicity. Note that all the ARPES data are measured at a photon energy of $150 \mathrm{eV}$ below $8 \mathrm{~K}$, and near the $Y-\Gamma-Y$ direction [see Fig. 3(d) for a precise measurement position]. Figure 1(c) shows the representative angle-integrated energy distribution curve (EDC), where spectral peaks can be identified by the (shallow) core levels of the constituent elements of YBCO $[14,15]$. By tuning the detection energy of photoelectrons to a specific core level and scanning spectral intensities on the sample surface, one can perform an element-selective spatial mapping.

Figure 1(f) displays the spatial distribution of the spectral weight near the Fermi level $\left(E_{\mathrm{F}}\right)$. Here, the intensity of each pixel represents the integrated spectral weight of each measured ARPES spectrum, resembling the ARPES images shown in Figs. 3(a) and 3(b), with limited energy integration [blue shaded area in Fig. 1(c)], while the momentum dimension was fully integrated. The pixel size corresponds to step sizes of these mappings that were set to be $50 \times 50 \mu \mathrm{m}^{2}$ so as to be comparable to the beam size. Importantly, the obtained spatial image clearly shows a significant intensity inhomogeneity, which should reflect electronic modulations on the surface. In contrast, the overall shape is almost identical to the optical image [Fig. 1(e)].

The next step is to identify the surface termination within the observed surface inhomogeneity. For this purpose, we utilized the $\mathrm{Ba} 4 d_{5 / 2}$ core level [red shaded area in Fig. 1(c)], considering the presence of two kinds of $\mathrm{BaO}$ - and $\mathrm{CuO}$-terminated surfaces in YBCO. Figure 2(a) shows the representative EDC spectra around the $\mathrm{Ba} 4 d_{5 / 2}$ core level taken at different sample positions, displaying a clear energy shift $(\sim 0.4 \mathrm{eV})$. The observed shift can be attributed to a chemical shift as it is significantly smaller than the reported surface core level shifts $(\sim 1 \mathrm{eV})$ of $\mathrm{Ba} 4 d$ core levels in YBCO [16]. Indeed, similar negative energy shifts in the $\mathrm{Ba} 4 d$ core levels have been observed by oxygen adsorption on a Ba surface [17]. Since such chemical variations can be expected to occur when the $\mathrm{Ba}$ layer is exposed on the topmost surface, the shallower (deeper) peak indicates that those measured positions is dominantly terminated by a $\mathrm{BaO}(\mathrm{CuO})$ layer. By limiting the energy integration windows as indicated in Fig. 2(a), we can examine the spatial distribution of the dominant region of the $\mathrm{CuO}$ and $\mathrm{BaO}$ layers as shown in Figs. 2(b) and 2(c). Furthermore, the 
(a)

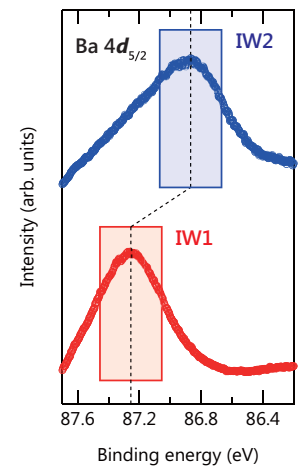

(b)

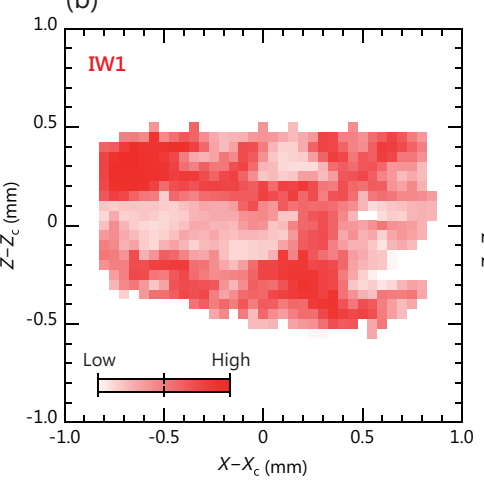

(c)

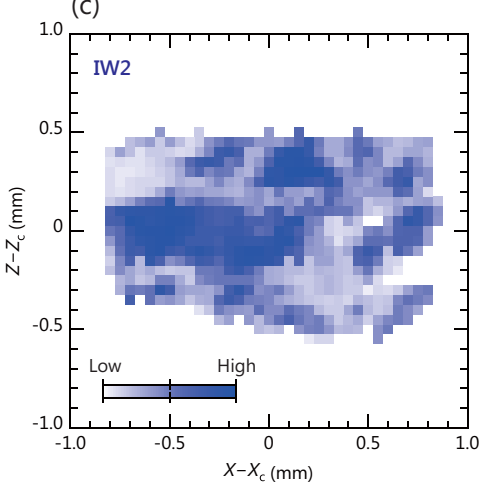

(d)

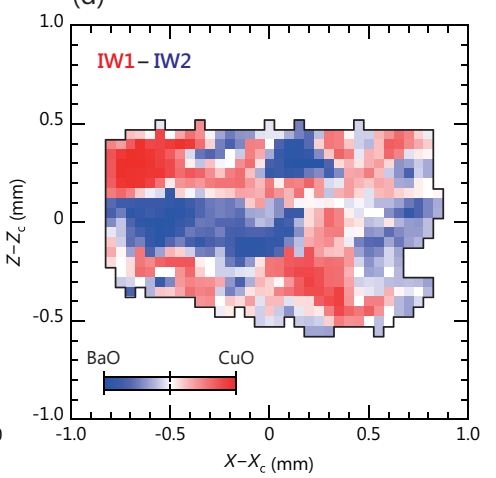

FIG. 2. Disentanglement of surface terminations of YBCO. (a) Representative Ba $4 d_{5 / 2}$ core-level spectra displaying chemical core-level shifts measured at different surface positions, where the spectral intensity was normalized by height. The red and blue shaded regions represent the integration windows (IW1 and IW2) used for mapping out the chemical-states-specified ARPES intensity distributions shown in (b) and (c), respectively. (d) Surface distribution of difference intensity between (b) and (c), where each spectral intensity of (b) and (c) is normalized by each averaged intensity. The sample surface is surrounded by the black line and the outer region should be ignored. These data are taken at a photon energy of $150 \mathrm{eV}$ below $7 \mathrm{~K}$.

spatial distribution of their intensity difference seen in Fig. 2(d) provides a clear identification of the dominant surface termination, enabling us to perform surface-termination-selective ARPES measurements.

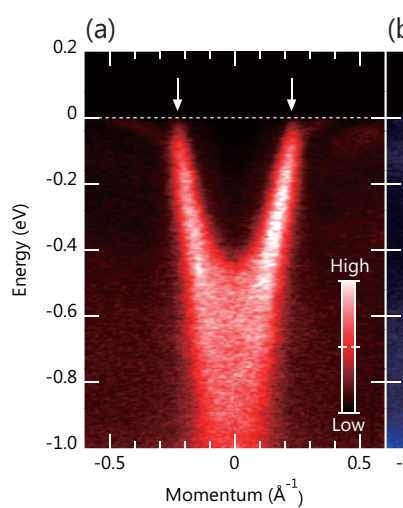

(b)
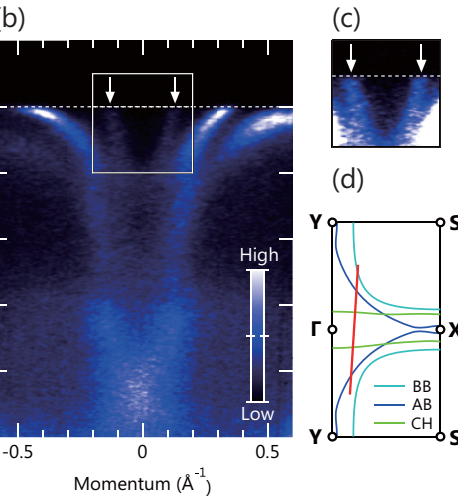

(d)

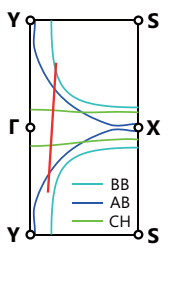

(e)
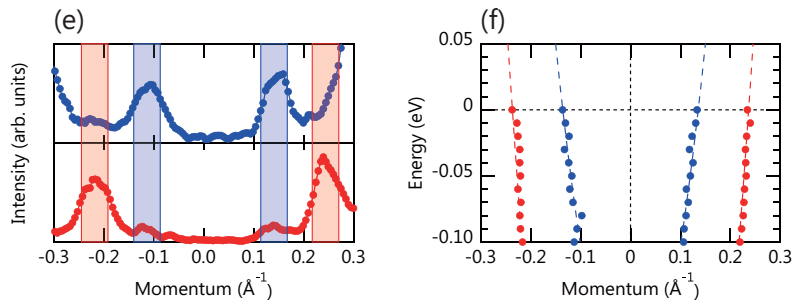

FIG. 3. Surface-termination-selective ARPES measurements on YBCO. (a) and (b) ARPES images taken from $\mathrm{CuO}$ - and $\mathrm{BaO}-$ terminated surfaces, respectively, measured at $150 \mathrm{eV}$ below $7 \mathrm{~K}$. (c) The $\mathrm{CuO}$-chain band on the $\mathrm{BaO}$-terminated surface, indicated by the white rectangle in (b), is highlighted by enhancing the color contrast. (d) Schematic Fermi surfaces of YBCO, where the red line indicates the measured momentum space of (a) and (b). (e) Momentum distribution curves (MDCs) integrated within $10 \mathrm{meV}$ of the Fermi level, from $\mathrm{CuO}$-terminated (lower) and $\mathrm{BaO}$-terminated surfaces (upper). (f) MDC-derived band dispersions of the dominant $\mathrm{CuO}$-chain bands [arrows in (a) and (b)] on the CuO-terminated surface (red) and $\mathrm{BaO}$-terminated surface (blue).
Having established the surface terminations, we have performed termination-dependent ARPES measurements. Figures 3(a) and 3(b) show the near- $E_{\mathrm{F}}$ ARPES images on the $\mathrm{CuO}$ - and $\mathrm{BaO}$-terminated surfaces, respectively, measured near the $Y-\Gamma-Y$ direction [red line in Fig. 3(d)]. As a natural expectation, ARPES signals from $\mathrm{CuO}$ - and $\mathrm{BaO}$-terminated surfaces are dominated by $\mathrm{CuO}$ chains and $\mathrm{CuO}_{2}$ planes below the $\mathrm{BaO}$ layer, respectively. Indeed, the most intense band in Fig. 3(a) is derived from the $\mathrm{CuO}$-chain band on the $\mathrm{CuO}$ terminated surface, while the two dominant bands in Fig. 3(b) arise from the bilayer-split $\mathrm{AB}$ and $\mathrm{BB}$ of the $\mathrm{CuO}_{2}$ planes [18]. At the same time, however, weak but finite signals were also detected besides intense features on both terminated surfaces. On the CuO-terminated surface, the $\mathrm{CuO}_{2}$-plane bands can be also observed in addition to the main $\mathrm{CuO}$-chain band in Fig. 3(a). Similarly, on the BaO-terminated surface, the $\mathrm{CuO}$-chain band can be recognized closer to the $\Gamma$ point inside the $\mathrm{CuO}_{2}$-plane bands in Fig. 3(b). This is more clearly shown in Fig. 3(c) by enhancing the color contrast.

By comparing the observed $\mathrm{CuO}$-chain bands on both surface terminations, we find clear differences in the momentum dispersion width as well as the Fermi momentum $\left(k_{\mathrm{F}}\right)$, as indicated by the arrows in Figs. 3(a) and 3(b). These observations manifest that the photoelectrons of the $\mathrm{CuO}$-chain band are emitted from different layers, respectively. Considering the layer stacking along the $c$ axis [Fig. 1(b)], the $\mathrm{CuO}$-chain states are thus inferred to be from the topmost $\mathrm{CuO}$-chain layer $\left(\mathrm{CH}_{\mathrm{TS}}\right)$ for the $\mathrm{CuO}$-terminated surface, while from the $\mathrm{CuO}$-chain layer underneath the topmost $\mathrm{CuO}_{2}$ bilayer and $\mathrm{BaO}$ block, namely, the subsurface $\mathrm{CuO}$-chain layer $\left(\mathrm{CH}_{\mathrm{SS}}\right)$, for the $\mathrm{BaO}$-terminated surface.

To investigate how two surface terminations are mixedly observed within a present probing area, we show the momentum distribution curves (MDCs) at $E_{\mathrm{F}}$ from the $\mathrm{CuO}$ - (red) and $\mathrm{BaO}$-terminated (blue) surfaces in Fig. 3(e). Prominent peaks in each curve can be attributed to the $\mathrm{CH}_{\mathrm{TS}}$ (red shaded area) and $\mathrm{CH}_{\mathrm{SS}}$ peaks (blue shaded area) for the $\mathrm{CuO}$ and $\mathrm{BaO}$-terminated surfaces, respectively, though these two states can be also observed on other types of termination. 
This demonstrates that small amounts of the $\mathrm{CuO}-(\mathrm{BaO}-)$ terminated surfaces are enclosed within the probing area for the dominant $\mathrm{BaO}-(\mathrm{CuO}-)$ terminated surface. Nevertheless, such in-plane termination mixing can be negligible judging from intensity ratios between majority and minority peaks due to the $\mathrm{CH}_{\mathrm{TS}}$ and $\mathrm{CH}_{\mathrm{SS}}$ or vice versa. Consequently, the photoemission signal on the $\mathrm{CuO}$ surface termination is dominated by the topmost $\mathrm{CuO}$-chain band and the subsurface $\mathrm{CuO}_{2}$-plane bands, and by the topmost $\mathrm{CuO}_{2}$-plane bands and the subsurface $\mathrm{CuO}$-chain band on the $\mathrm{BaO}$ surface termination.

Figure 3(f) shows the band dispersions of the $\mathrm{CH}_{\mathrm{TS}}$ (red) and $\mathrm{CH}_{\mathrm{SS}}$ (blue) states obtained by fitting MDCs taken from the $\mathrm{CuO}-$ and $\mathrm{BaO}$-terminated surfaces, respectively. We evaluate the band parameters by fitting the MDC dispersion to a quadratic function $\varepsilon_{k}=-\varepsilon_{0}^{*}+\left(\varepsilon_{0}^{*} / k_{\mathrm{F}}^{2}\right) k^{2}$, where $\varepsilon_{0}^{*}$ is a renormalized energy of a band bottom. The red and blue dashed lines represent the obtained quadratic functions for the $\mathrm{CH}_{\mathrm{TS}}$ and $\mathrm{CH}_{\mathrm{SS}}$ states with the following parameters: $k_{\mathrm{F}}=$ $0.236 \pm 0.001 \AA, \varepsilon_{0}^{*}=0.65 \pm 0.01 \mathrm{eV}$ for the $\mathrm{CH}_{\mathrm{TS}}$ for the $\mathrm{CuO}$-terminated surface, while $k_{\mathrm{F}}=0.135 \pm 0.002 \AA, \varepsilon_{0}^{*}=$ $0.22 \pm 0.02 \mathrm{eV}$ for the $\mathrm{CH}_{\mathrm{SS}}$ for the $\mathrm{BaO}$-terminated surface. Then, the effective mass, $m^{*}=\hbar^{2} /\left(\partial^{2} \varepsilon_{k} / \partial k^{2}\right)=\hbar^{2} k_{\mathrm{F}}^{2} / 2 \varepsilon_{0}^{*}$, can be estimated as $0.33 \pm 0.01 m_{e}$ and $0.32 \pm 0.03 m_{e}$ for the $\mathrm{CuO}-$ and $\mathrm{BaO}$-terminated surfaces, respectively, where $m_{e}$ is the electron mass. These results are indicative of two important issues. First, the observed two $\mathrm{CH}_{\mathrm{TS}}$ and $\mathrm{CH}_{\mathrm{SS}}$ states follow a simple rigid-band model with different electron fillings. Second, they show the presence of electronlike carriers with a high mobility on the $\mathrm{CuO}$ chains, which can account for the negative Hall resistivity in YBCO [19].

Note that these two different CuO-chain states were previously reported using a soft x-ray ARPES [20], where the outer CuO-chain band with a larger $2 k_{\mathrm{F}}=0.55 \AA^{-1}$ was observed for the first time accompanied by a pseudogap of an energy scale of 200-300 meV. As has been already demonstrated, the reported outer $\mathrm{CuO}$-chain band corresponds to the $\mathrm{CH}_{\mathrm{TS}}$ states on the CuO-terminated surface. Moreover, since the observed $\mathrm{CH}_{\mathrm{TS}}$ states are apparently crossing $E_{\mathrm{F}}$, the spectral weight suppression reported in Ref. [20] is probably not related to the pseudogap phenomena but rather an effect of the matrix element and/or $k_{z}$ dispersion, both of which are dependent on photon energy. Although one may consider that the quasi-one-dimensional $\mathrm{CuO}$-chain band should not show a $k_{z}$ dependence, a recent $\mathrm{x}$-ray absorption spectroscopy (XAS) and resonant inelastic $\mathrm{x}$-ray scattering (RIXS) study on optimally doped $\mathrm{YBCO}$ has revealed a charge transfer between the $\mathrm{CuO}$ chains and the $\mathrm{CuO}_{2}$ planes [21]. Hence, the $k_{z}$ dispersion of the $\mathrm{CuO}$-chain band can be generated by orbital hybridization between the $\mathrm{CuO}$-chain $3 d_{y^{2}-z^{2}}$ orbitals and $\mathrm{CuO}_{2}$-plane $3 d_{3 z^{2}-r^{2}}$ orbitals via apical oxygen $2 p_{z}$ orbitals.

Led by the observation of different electron dopings to the $\mathrm{CuO}$ chain between the $\mathrm{CuO}-$ and $\mathrm{BaO}$-terminated surfaces, we further examine quantitatively the doping levels based on the Fermi-surface mapping. Figures 4(a) and 4(b) show the Fermi-surface maps on the same $\mathrm{BaO}$-terminated surface with $\pm 10 \mathrm{meV}$ energy integration, which were measured by azimuthal rotations of $90^{\circ}$ in situ. The determined $k_{\mathrm{F}}$ values for the $\mathrm{BB}, \mathrm{AB}$, and $\mathrm{CH}_{\mathrm{SS}}$ are summarized in Fig. 4(c), where (a)

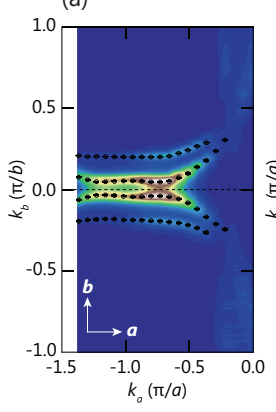

(d)

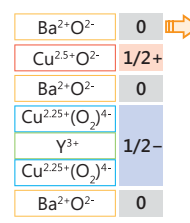

(b)

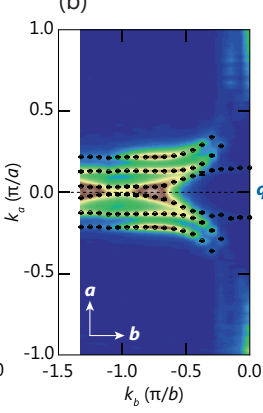

(c)

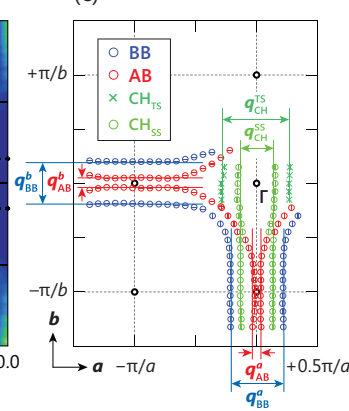

(e)
FIG. 4. Determination of doping levels of the cleaved YBCO surface. (a) and (b) Constant energy maps of ARPES intensity integrated within $10 \mathrm{meV}$ of the Fermi level $\left(E_{\mathrm{F}}\right)$, measured at $50 \mathrm{eV}$ below $9 \mathrm{~K}$ with two different sample orientations. The detection of photoelectrons was performed in parallel to the crystal $b$ axis and $a$ axis in (a) and (b), respectively. (c) Fermi momentum plots for the bonding and antibonding $\mathrm{CuO}_{2}$-plane bands ( $\mathrm{BB}$ and $\mathrm{AB}$ ) and the $\mathrm{CuO}$-chain band on the topmost surface and subsurface $\left(\mathrm{CH}_{\mathrm{TS}}\right.$ and $\mathrm{CH}_{\mathrm{SS}}$ ), determined by fitting momentum distribution curves (MDCs) at the $E_{\mathrm{F}}$ with $\pm 10 \mathrm{meV}$ integration. Model electronic configurations before (left) and after (right) cleaving for (d) $\mathrm{CuO}$-terminated surfaces and (e) $\mathrm{BaO}$-terminated surfaces.

the $\mathrm{CH}_{\mathrm{TS}}$ are also plotted but only partially because the reliable region is limited due to severe merging against the $\mathrm{CuO}_{2}$-plane bands near the antinodal region.

By fitting a tight-binding model to the Fermi surfaces, the CuO-chain Fermi-surface area relative to the $\mathrm{BZ}$ area $S=4 \pi^{2} / a b$ was determined to be $S=24.9 \%$ and $15.2 \%$ for the $\mathrm{CuO}$ - and $\mathrm{BaO}$-terminated surfaces, respectively. The corresponding numbers of electrons $n$ are thus counted as 0.48 and 0.27 for $\mathrm{CuO}$ - and $\mathrm{BaO}$-terminated surfaces, reconfirming the hole-rich states on the latter surface.

On the other hand, the $\mathrm{CuO}_{2}$-plane Fermi-surface area was determined directly from the $k_{\mathrm{F}}$ plots of $\mathrm{BB}$ and $\mathrm{AB}$ on the $\mathrm{BaO}$-terminated surface to be $S_{\mathrm{BB}}=40.9 \%$ and $S_{\mathrm{AB}}=19.5 \%$. By taking the average of bonding and antibonding Fermisurface areas, the hole doping level of the $\mathrm{CuO}_{2}$ planes can be calculated as $p=0.40$ per planar copper $(p=0$ for 1 hole per $\mathrm{Cu}$ atom). Note that such heavy overdoping of the $\mathrm{CuO}_{2}$-plane bands is qualitatively consistent with the reported values $p \sim 0.30-0.37[3,4,11]$.

Summarizing the above observations, the number of holes in the $\mathrm{CuO}$ chains $(\eta)$ and $\mathrm{CuO}_{2}$ planes $(\zeta)$ are $\eta=1.73$ and $\zeta=1.40$ for the $\mathrm{BaO}$-terminated surface, and $\eta=1.52$ for the $\mathrm{CuO}$-terminated surface. Here, we focus on and discuss the surface electronic reconstruction in a stoichiometric composition $\mathrm{YBa}_{2} \mathrm{Cu}_{3} \mathrm{O}_{7}$ (YBCO7) near the present optimal doping [22]. Kamimura and Sano [23] proposed feasible model electronic configurations in YBCO7, in which the average charges are $+2.5 e$ in $\mathrm{Cu}(1)$-chain sites and $+2.25 e$ in $\mathrm{Cu}(2)$-plane sites ( 1.5 holes per $\mathrm{CuO}$ chain and $1 / 4$ hole per $\mathrm{CuO}_{2}$ plane). 
TABLE I. Extracted wave vector $q=2 k_{\mathrm{F}}$ as indicated in Fig. 4(c) and corresponding wavelength $\lambda$ calculated by $2 \pi / q$ for the $\mathrm{CuO}$ chain band $(\mathrm{CH})$ on the topmost surface (TS) and subsurface (SS), and the bonding and antibonding bands of the $\mathrm{CuO}_{2}$ plane $(\mathrm{BB}$ and $\mathrm{AB})$ along each $a$ and $b$ axis.

\begin{tabular}{lccccrr}
\hline \hline & $q_{\mathrm{CH}}^{\mathrm{TS}}$ & $q_{\mathrm{CH}}^{\mathrm{SS}}$ & $q_{\mathrm{BB}}^{a}$ & $q_{\mathrm{AB}}^{a}$ & $q_{\mathrm{BB}}^{b}$ & $q_{\mathrm{AB}}^{b}$ \\
\hline $2 k_{\mathrm{F}}\left(\AA^{-1}\right)$ & 0.48 & 0.25 & 0.36 & 0.04 & 0.31 & 0.08 \\
$\lambda(\AA)$ & 13.1 & 25.1 & 17.5 & 157.1 & 20.27 & 78.54 \\
\hline \hline
\end{tabular}

Note that the model holds the condition of an overall charge neutrality, $\eta+2 \zeta=2$, while $\eta$ and $\zeta$ are interrelated through the charge-density-wave (CDW) modulation with the most plausible CDW wavelength $q_{\mathrm{CDW}}=2 \pi / 2 k_{\mathrm{F}}=4 b$ consistent with STM [24,25] and neutron diffraction [26].

Comparing the ARPES results to the model, we find a close resemblance of the $\eta$ in the $\mathrm{Cu}(1)$ chains for the CuO-terminated surface, while clear deviations of the $\eta$ and $\zeta$ in the $\mathrm{Cu}(1)$ chains and $\mathrm{Cu}(2)$ planes for the $\mathrm{BaO}$ terminated surface. This strongly suggests a terminationdependent surface electronic reconstruction, namely, the electronic configurations are unreconstructed on the $\mathrm{CuO}$ terminated surface, while reconstructed on the $\mathrm{BaO}$-terminated surface.

Previously, the surface reconstruction in the YBCO system has been considered as originating from the so-called polar catastrophe model [10,27]. The polar catastrophe model is well known for explaining the emergence of a two-dimensional electron gas (2DEG) in the interfaces of a two-insulating-oxide $\mathrm{LaAlO}_{3} / \mathrm{SrTiO}_{3}$ heterostructure [28]. The heart of this model is a transfer of net charges across a polar interface. This interfacial reconstruction is required to avoid a diverging electric potential due to a nonzero electric field produced by layer-by-layer alternating net charges as $|1+| 1-|\cdots| 1+|1-|$.

Whereas such charge distributions of $|1 / 2+| 1 / 2-\mid$ are realized between the $\mathrm{CuO}$-chain and $\mathrm{CuO}_{2}$-plane $\left(\mathrm{CuO}_{2}-\mathrm{Y}-\mathrm{CuO}_{2}\right)$ blocks based on the above-mentioned model configuration in $\mathrm{YBCO} 7$, the polar catastrophe model cannot explain the here observed charge distributions as it predicts a charge transfer for both surface terminations; a $1 / 4$ electron is transferred into the $\mathrm{CuO}$-terminated surface, while a $1 / 4$ hole into the BaO-terminated surface (see Fig. S1 of the Supplemental Material [29]). On the other hand, recent first-principles density functional theory calculations on $\mathrm{LaAlO}_{3} / \mathrm{SrTiO}_{3}$ systems pointed out the possibility of the absence of electronic reconstruction even though alternating $|1+| 1-\mid$ charges are realized in the case of a stoichiometric $1 / 3 \mathrm{LaAlO}_{3} / \mathrm{SrTiO}_{3}$ nanowire due to a smaller effective concentration of $\mathrm{LaAlO}_{3}$ on the surface [30]. In this context, there still remains the possibility that a modified polar catastrophe model might explain the observed electronic construction. However, assuming the relevance of the polarity for the observed electronic reconstruction in YBCO, this would correspond to a very special situation that may be verified by realistic model computations considering the in-plane domain sizes as well as the actual $c$-axis stacking involving nonpolar $\mathrm{BaO}$ layers between the $\mathrm{CuO}$ chains and $\mathrm{CuO}_{2}$ planes.

Alternatively, we propose a phenomenological model for the electronic configurations for the $\mathrm{CuO}$ - and $\mathrm{BaO}$-terminated surfaces as shown in Figs. 4(d) and 4(e), respectively. In the case of the CuO-terminated surface [Fig. 4(d)], the charge balance is unaffected across cleaving (left to right), resulting in no net charge transfer. In contrast, in the case of the BaO-terminated surface [Fig. 4(e)], a charge imbalance with respect to the topmost $\mathrm{BaO}$ layer is generated. The removed charge of $1 / 2+$ is then compensated from neighboring atoms to maintain an overall charge neutrality, where we presume the range of this compensation to be within one unit cell both in plane as well as out of plane. Assuming a uniform charge redistribution into the $\mathrm{CuO}_{2}$ planes and subsurface $\mathrm{CuO}$ chains in the $\mathrm{BaO}$-terminated surface, the average charges become $+2.375 e$ in the $\mathrm{Cu}(2)$-plane sites with $\zeta=1.375$, and $+2.75 e$ in the $\mathrm{Cu}(1)$-chain sites with $\eta=1.75$, which is quantitatively consistent with the present ARPES results $(\zeta=1.40, \eta=1.73)$.

The present model is also supported by the wavelength $\lambda=$ $2 \pi / q$ corresponding to $q=2 k_{\mathrm{F}}$ as extracted from Fig. 4(c). As summarized in Table $\mathrm{I}$, the $\lambda$ of the topmost $\mathrm{CuO}$ chain states $\mathrm{CH}_{\mathrm{TS}}$ only exactly agrees with the periodicity of $13 \AA$ reported in STM experiments [24,25], while the others show a substantial discrepancy. This validates our interpretation of the unreconstructed electronic configurations with the CDW modulation for the $\mathrm{CuO}$-terminated surface, while surface electronic reconstructions occur and the periodicity is consequently disturbed for the $\mathrm{BaO}$-terminated surface. The present results imply that the nominal doping level of the $\mathrm{CuO}_{2}$ planes remains for the CuO-terminated surface, which should be responsible for the bulklike components observed in previous ARPES studies [2-5].

In summary, we have revealed the surface inhomogeneity of optimally doped YBCO by spatially resolved microARPES. The spatial distribution of surface terminations was identified based on the chemical and electronic information. Termination-selective ARPES measurements reveal two distinct $\mathrm{CuO}$-chain bands with different charge fillings, indicating the occurrence of termination-dependent electronic reconstructions. By combining the spatial and electronic information, we proposed a simple model to explain the charge distributions both for unreconstructed topmost $\mathrm{CuO}$-chain bands on the CuO-terminated surface as well as reconstructed $\mathrm{CuO}_{2}$ plane and subsurface $\mathrm{CuO}$-chain bands in the $\mathrm{BaO}$-terminated surface.

We thank Hirofumi Kawanaka and Yoshihiro Aiura for superconducting quantum interference device (SQUID) remeasurements. We thank Diamond Light Source for access to beamline I05 (Proposals No. NT16871 and No. NT17192) that contributed to the results presented here. 
[1] H. L. Edwards, J. T. Markert, and A. L. de Lozanne, Phys. Rev. Lett. 69, 2967 (1992).

[2] K. Nakayama, T. Sato, K. Terashima, H. Matsui, T. Takahashi, M. Kubota, K. Ono, T. Nishizaki, Y. Takahashi, and N. Kobayashi, Phys. Rev. B 75, 014513 (2007).

[3] V. B. Zabolotnyy, S. V. Borisenko, A. A. Kordyuk, J. Geck, D. S. Inosov, A. Koitzsch, J. Fink, M. Knupfer, B. Büchner, S.-L. Drechsler, H. Berger, A. Erb, M. Lambacher, L. Patthey, V. Hinkov, and B. Keimer, Phys. Rev. B 76, 064519 (2007).

[4] K. Nakayama, T. Sato, K. Terashima, T. Arakane, T. Takahashi, M. Kubota, K. Ono, T. Nishizaki, Y. Takahashi, and N. Kobayashi, Phys. Rev. B 79, 140503(R) (2009).

[5] M. Okawa, K. Ishizaka, H. Uchiyama, H. Tadatomo, T. Masui, S. Tajima, X.-Y. Wang, C.-T. Chen, S. Watanabe, A. Chainani, T. Saitoh, and S. Shin, Phys. Rev. B 79, 144528 (2009).

[6] M. C. Schabel, C.-H. Park, A. Matsuura, Z.-X. Shen, D. A. Bonn, X. Liang, and W. N. Hardy, Phys. Rev. B 57, 6090 (1998).

[7] D. H. Lu, D. L. Feng, N. P. Armitage, K. M. Shen, A. Damascelli, C. Kim, F. Ronning, and Z.-X. Shen, D. A. Bonn, R. Liang, W. N. Hardy, A. I. Rykov, and S. Tajima, Phys. Rev. Lett. 86, 4370 (2001).

[8] A. Williams, G. H. Kwei, R. B. Von Dreele, A. C. Larson, I. D. Raistrick, and D. L. Bish, Phys. Rev. B 37, 7960(R) (1988).

[9] M. P. Seah and W. A. Dench, Surf. Interface Anal. 1, 2 (1979).

[10] M. A. Hossain, J. D. F. Mottershead, D. Fournier, A. Bostwick, J. L. McChesney, J. L. McChesney, E. Rotenberg, R. Liang, W. N. Hardy, G. A. Sawatzky, I. S. Elfimov, D. A. Bonn, and A. Damascelli, Nat. Phys. 4, 527 (2008).

[11] D. Fournier, G. Levy, Y. Pennec, J. L. McChesney, A. Bostwick, E. Rotenberg, R. Liang, W. N. Hardy, D. A. Bonn, I. S. Elfimov, and A. Damascelli, Nat. Phys. 6, 905 (2010).

[12] Y. Yamada and Y. Shiohara, Physica C 217, 182 (1993).

[13] M. Hoesch, T. K. Kim, P. Dudin, H. Wang, S. Scott, P. Harris, S. Patel, M. Matthews, D. Hawkins, S. G. Alcock, T. Richter, J. J. Mudd, M. Basham, L. Pratt, P. Leicester, E. C. Longhi, A. Tamai, and F. Baumberger, Rev. Sci. Instrum. 88, 013106 (2017).

[14] T. Sebastian, Interface structure and electronic properties of $\mathrm{SrTiO}_{3}$ and $\mathrm{YBa}_{2} \mathrm{Cu}_{3} \mathrm{O}_{7-\delta}$ crystals and thin films, Ph.D. thesis, University of Hamburg, 2007.

[15] S. Thiess, T.-L. Lee, C. Aruta, C. T. Lin, F. Venturini, N. B. Brookes, B. C. C. Cowie, and J. Zegenhagen, Phys. Rev. B 92, 075117 (2015).
[16] K. Maiti, J. Fink, S. de Jong, M. Gorgoi, C. Lin, M. Raichle, V. Hinkov, M. Lambacher, A. Erb, and M. S. Golden, Phys. Rev. B 80, 165132 (2009).

[17] D. Vlachos, M. Kamaratos, and S. D. Foulias, J. Phys.: Condens. Matter 18, 6997 (2006).

[18] S. V. Borisenko, A. A. Kordyuk, V. Zabolotnyy, J. Geck, D. Inosov, A. Koitzsch, J. Fink, M. Knupfer, B. Büchner, V. Hinkov, C. T. Lin, B. Keimer, T. Wolf, S. G. Chiuzbăian, L. Patthey, and R. Follath, Phys. Rev. Lett. 96, 117004 (2006).

[19] D. LeBoeuf, Ni. Doiron-Leyraud, J. Levallois, R. Daou, J.-B. Bonnemaison, N. E. Hussey, L. Balicas, B. J. Ramshaw, R. Liang, D. A. Bonn, W. N. Hardy, S. Adachi, C. Proust, and L. Taillefer, Nature (London) 450, 533 (2007).

[20] V. B. Zabolotnyy, A. A. Kordyuk, D. Evtushinsky, V. N. Strocov, L. Patthey, T. Schmitt, D. Haug, C. T. Lin, V. Hinkov, B. Keimer, B. Büchner, and S. V. Borisenko, Phys. Rev. B 85, 064507 (2012).

[21] M. Magnuson, T. Schmitt, V. N. Strocov, J. Schlappa, A. S. Kalabukhov, and L.-C. Duda, Sci. Rep. 4, 7017 (2014).

[22] Strictly speaking, the present optimally doped YBCO6.9 $(\delta=$ $0.1)$ has a different composition from the stoichiometric YBCO7 ( $\delta=0.0)$, but the expected doping difference between them is 0.02 in the unit cell [R. Liang, D. A. Bonn, and W. N. Hardy, Phys. Rev. B 73, 180505(R) (2006)]; since the difference is considerably small compared with the observed charge reconstruction, it is negligible in the discussions and does not affect our conclusions.

[23] H. Kamimura and A. Sano, Solid State Commun. 109, 543 (1999).

[24] H. L. Edwards, A. L. Barr, J. T. Markert, and A. L. de Lozanne, Phys. Rev. Lett. 73, 1154 (1994).

[25] M. Maki, T. Nishizaki, K. Shibata, and N. Kobayashi, Phys. Rev. B 65, 140511(R) (2002).

[26] H. A. Mook, P. Dai, K. Salama, D. Lee, F. Doğan, G. Aeppli, A. T. Boothroyd, and M. E. Mostoller, Phys. Rev. Lett. 77, 370 (1996).

[27] K. Pasanai and W. A. Atkinson, Phys. Rev. B 81, 134501 (2010).

[28] N. Nakagawa, H. Y. Hwang, and D. A. Muller, Nat. Mater. 5, 204 (2006).

[29] See Supplemental Material at http://link.aps.org/supplemental/ 10.1103/PhysRevB.98.081112 for modeling a polar catastrophe against the $\mathrm{CuO}$ - and $\mathrm{BaO}$-terminated surfaces of the optimally doped YBCO.

[30] H. L. Zhuang, L. Zhang, H. Xu, P. R. C. Kent, P. Ganesh, and V. R. Cooper, Sci. Rep. 6, 25452 (2016). 